\title{
Ligand Size Distribution of Phenanthroline- Functionalized Polyethylene Glycol-Iron(II) Complexes Determined by Electrospray Ionization Mass Spectrometry and Computer Simulation
}

\author{
Ákos Kuki, Miklós Nagy, Lajos Nagy, Miklós Zsuga, and Sándor Kéki \\ Department of Applied Chemistry, University of Debrecen, Debrecen, Hungary
}

\begin{abstract}
The complex formation in solution, and the gas-phase dissociation of a phenanthrolineterminated poly(ethylene glycol) with $\mathrm{Fe}^{2+}$ ions were investigated. The size distribution of poly(ethylene glycol)- $\alpha$-monomethyl- $\omega$-5-[1,10]phenanthroline (mPEG_phen) was determined by electrospray ionization mass spectrometry (ESI-MS). Based on the measured ligand size distribution of mPEG_phen by ESI-MS, the 1:3 complex formation ( $\mathrm{Fe}^{2+} / \mathrm{mPEG}$ Phen) was computer-simulated as a pure random assembly process. The simulated distribution fits excellently to that of the complex Fe(mPEG_phen) ${ }_{3}^{2+}$ determined from the ESI-MS intensities. In addition, the collision-induced dissociation (CID) of the Fe(mPEG_phen) ${ }_{3}^{2+}$ complex was also studied by tandem mass spectrometry (ESI-MS/MS) and by computer simulation, as well. The ESI-MS/MS intensity distribution of the Fe(mPEG_phen $)_{2}^{2+}$ formed from Fe(mPEG_ phen $)_{3}^{2+}$ by the loss of an mPEG_phen ligand under CID conditions fits quite well to the calculated one. (J Am Soc Mass Spectrom 2010, 21, 1561-1564) (C) 2010 American Society for Mass Spectrometry
\end{abstract}

$\mathrm{E}$ lectrospray ionization mass spectrometry (ESIMS) [1] offers a unique tool for the investigation of metal-containing complexes because it overcomes the propensity of these complex ions to dissociate when transferred from solution into the gas phase [2-8]. In addition, the gas-phase complex ions of interest can then be selected and subjected to collisioninduced dissociation (CID) to promote the detachment of the intact ligands. In this way, the determination of the relative gas-phase stability of the different complex ions is feasible as it has been shown in several papers [6-9]. Although various types of complexes have been used in several ESI-MS studies, to our knowledge, no ESI-MS report on complexes with ligands consisting of polymer chains has appeared. Since a real polymer is composed of polymer chains with varying chain lengths, it is interesting to study the effect of the chain length on the complex formation and dissociation of these complexes in the gas phase by utilizing the capability of ESI-MS.

In this paper, we report the ESI-MS and computer simulation studies of the complex formation of a phenanthroline-functionalized polyethylene glycol

Address reprint requests to Dr. S. Kéki, Department of Applied Chemistry, University of Debrecen, Egyetem ter. 1, Debrecen, Hungary. E-mail: keki@tigris.unideb.hu with $\mathrm{Fe}^{2+}$ ion and the dissociation of this complex under CID conditions.

\section{Experimental}

\section{Materials}

HPLC grade methanol (Scharlau, Sentmenat, Spain), Mohr's salt (Spektrum 3D, Debrecen, Hungary), sodium trifluoroacetate, and 5,6-epoxy-5,6-dihydro- [1,10]phenanthroline (Sigma-Aldrich, Steinheim, Germany) were used without further purification. Poly(ethylene glycol) 1100 monomethyl ether was purchased from Fluka (Buchs, Switzerland). The structure of poly(ethylene glycol)- $\alpha$ monomethyl- $\omega-5-[1,10]$ phenanthroline ether (mPEG_ phen) is presented in Scheme 1. The detailed synthesis of mPEG_phen will be described in a separate paper.

\section{Methods}

Electrospray quadrupole time-of-flight mass spectrometry (ESI-QTOF MS). Measurements were performed with a MicroTOF-Q-type Qq-TOF MS instrument equipped with an ESI source (Bruker Daltonics, Bremen, Germany). The solutions at a concentration of $10 \mu \mathrm{M}$ were introduced directly into the ESI source with a syringe pump (Cole-Parmer Ins. Comp., Vernon Hills, IL, USA) at a $3 \mu \mathrm{L} / \mathrm{min}$ flow rate. The Fe(mPEG_phen) ${ }_{3}^{2+}$ com- 
<smiles>CCC(C)(C)OCCOC(C)(C)C</smiles>

Scheme 1. The structure of poly(ethylene glycol)- $\alpha$-monomethyl$\omega-5-[1,10]$ phenanthroline ether (mPEG_phen)

plex was prepared from Mohr's salt and mPEG_phen in 1:3 ( $\mathrm{Fe}^{2+} /$ ligand) molar ratio and diluted with methanol to obtain a $10 \mu \mathrm{M}$ solution. The temperature of the drying gas $\left(\mathrm{N}_{2}\right)$ was maintained at $160^{\circ} \mathrm{C}$. The needle voltage was kept at $4 \mathrm{kV}$. For CID experiments $\mathrm{N}_{2}$ was used as the collision gas at a pressure of $8 \times 10^{-3}$ mbar. The precursor ions of interest were selected with a mass window of 6 Daltons and collision voltages of 60-93 V were applied. All the mass spectra were calibrated externally using the exact masses of clusters [(NaTFA $)_{n}$ $+\mathrm{Na}]^{+}$generated from the electrosprayed solution of sodium trifluoroacetate (NaTFA). The mass spectra recorded were evaluated by the DataAnalysis 3.1 software from Bruker.

Computer simulation. The simulation of the complex formation is given in the Supplemental Information (Algorithm 1), which can be found in the electronic version of this article. The ligands array contains the ligand length values, each element represents a ligand. Three ligands are chosen from this array randomly to form a complex. The consumed ligands are deleted form the ligands array. After assembling a complex, the appropriate element of the complexes counter array is incremented.

The computer simulation procedure of the fragmentation process of the PEG-phenanthroline-iron(II) complex ions is presented in the Supplemental Information (Algorithm 2). The complexes with 84 ethylene oxide repeat units are chosen and their lengths are decreased with the length of a randomly selected ligand. Then the appropriate element of the fragmented_complexes counter array is incremented. Two consecutive ligand losses can be simulated by a slight modification of Algorithm 2.

\section{Results and Discussion}

Our preliminary goal was to study the relationship between the ESI-MS intensity of poly(ethylene glycol)$\alpha$-monomethyl- $\omega$-5-[1,10]phenanthroline ether (mPEG_ phen) and that of the Fe(mPEG_phen) ${ }_{3}^{2+}$ complex. Figure 1 shows the normalized distribution of the ligand mPEG_phen as a function of the number of repeat units (ethylene oxide) as determined from the corresponding ESI-MS intensities.

As seen in Figure 1, mPEG_phen is composed of polymer chains with varying number of repeat units (n)

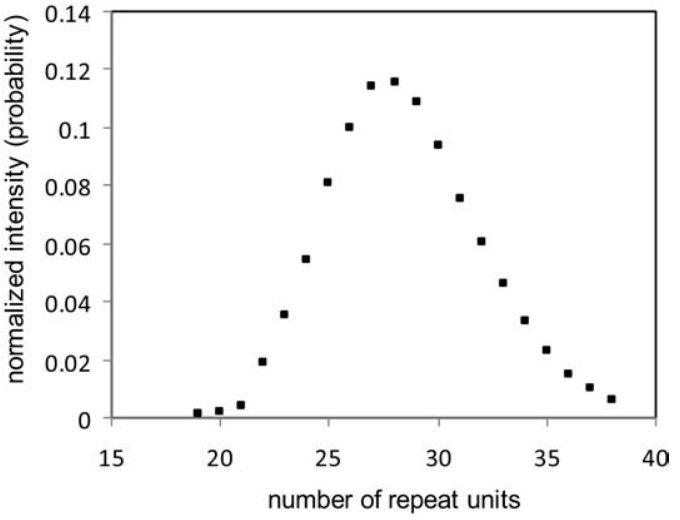

Figure 1. The normalized intensity distribution of the mPEG phen as a function of the number of repeat units. The normalized intensities $\left(\mathrm{I}_{\text {norm }, i}\right)$ were obtained as: $\mathrm{I}_{\text {norm }, i}=\mathrm{I}_{i} / \sum \mathrm{I}_{i}$ where $\mathrm{I}_{i}$ is the ESI-MS intensity of mPEG_phen with a number of repeat units $i$ and $\Sigma I_{i}$ is the sum of all mPEG_phen intensities. (The ESI-MS signals are originated from the sodiated oligomers). Note that this normalized intensity distribution expresses also the probability of finding mPEG_phen chain with a number of repeat unit $i$.

that $\operatorname{span}$ from $n=19$ to $n=38$ and centered at $n=28$. In the next experiment a tris-complex of $\mathrm{Fe}^{2+}$ was prepared using the mPEG_phen ligand whose distribution is presented in Figure 1. The ESI-MS spectrum of the tris-complex of $\mathrm{Fe}^{2+}$ is shown in Figure 2.

As Figure 2 shows, the tris-complex occurs with different charge states in the ESI-MS spectrum. For example, doubly charged ions originate from the complex Fe(mPEG_phen) $)_{3}^{2+}$, while the triply and quadruply charged ones are due to the additional attachment of one and two $\mathrm{NH}_{4}^{+}$ions, respectively, to the PEG chains of the tris-complex. The presence of $\mathrm{NH}_{4}^{+}$ions are due

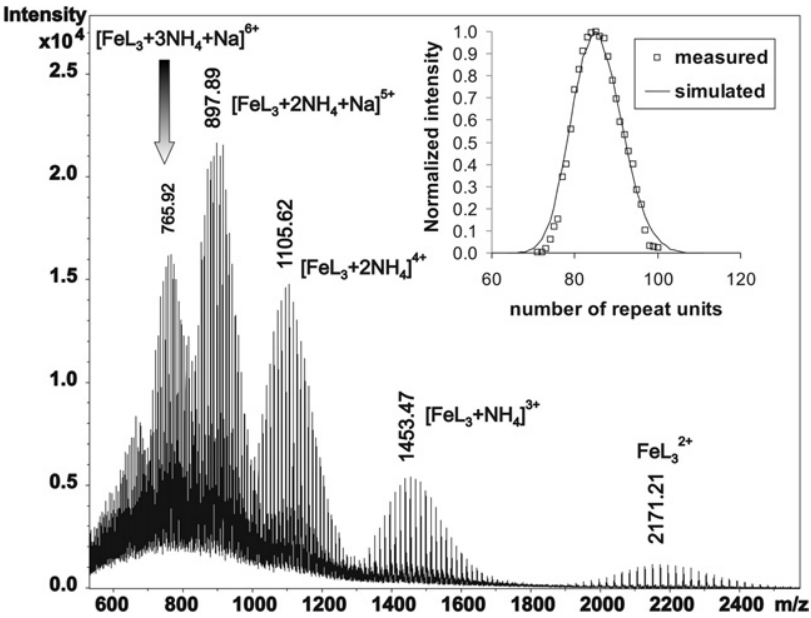

Figure 2. ESI-MS spectrum of the Fe(mPEG_phen $)_{3}^{2+}$ complex. The inset shows the measured and the calculated normalized intensity distributions for the Fe(mPEG_phen $)_{3}^{2+}$ as a function of the total number of repeat units. L stands for the mPEG_phen ligand. The normalized intensities $\left(\mathrm{I}_{\text {norm }, i}\right)$ were obtained as: $\mathrm{I}_{\text {norm }, i}=\sum_{j} \mathrm{I}_{i, 1} \mathrm{I}_{\max }$ where $\sum_{j} \mathrm{I}_{i, j}$ is the sum of intensities of Fe(mPEG_phen $)_{3}^{2+}$ containing a total number of repeat units $i$ in charge states $(j)$ ranging from +2 to $+6 . I_{\max }$ is the maximum intensity obtained after summation. 
to the Mohr's salt used for the preparation of the $\mathrm{Fe}(\text { mPEG_phen })_{3}^{2+}$ complex, as it was shown that $\mathrm{NH}_{4}^{+}$ is able to attach to the polyether chains [10]. In the following, we determined the intensity distribution for the $\mathrm{Fe}\left(\mathrm{mPEG}_{\mathrm{P}}\right.$ phen ${ }_{3}^{2+}$ complexes from the ESI-MS spectrum as a function of the total number of the repeat units. To obtain the intensity distribution of the $\mathrm{Fe}(\mathrm{m}$ PEG_phen) ${ }_{3}^{2+}$ complexes, the ESI-MS intensities at different charge states were summed and then compared with the distribution calculated on the basis of the procedure to be presented below. It is to be noted, however, that consecutive additions of the intensities of charge states from +3 to +6 to those of charge state +2 does not significantly affect the resulting intensity distribution (see the Supplemental Information).

The formation of the Fe(mPEG_phen) ${ }_{3}^{2+}$ complexes can be given by eq 1 ,

$$
\mathrm{Fe}^{2+}+\mathrm{L}_{\mathrm{x}}+\mathrm{L}_{\mathrm{y}}+\mathrm{L}_{\mathrm{z}}=\left[\mathrm{FeL}_{\mathrm{x}} \mathrm{L}_{\mathrm{y}} \mathrm{L}_{\mathrm{z}}\right]^{2+}
$$

where $L$ is the ligand, mPEG_phen and the subscripts represent the numbers of the repeat units.

To calculate the expected intensity distribution for $\mathrm{Fe}\left(\right.$ mPEG_phen $_{3}^{2+}$ as a function of the total number of repeat units, i.e., $\mathrm{x}+\mathrm{y}+\mathrm{z}$ according to eq 1 , we applied a short computer program presented in the Supplemental Information. Our program worked with $\mathrm{mPEG}_{-}$ phen particle quantity of 660,000 distributed according to that shown in Figure 1.

Three mPEG_phen particles were chosen randomly to form a Fe(mPEG_phen) $)_{3}^{2+}$ complex ion. All of the mPEG_phen was consumed during the simulation process, so $220,000 \mathrm{Fe}\left(\mathrm{mPEG}\right.$ _phen ${ }_{3}^{2+}$ complex ions were formed.

Figure 2 inset shows the measured and the calculated intensity distributions for the $\mathrm{Fe}\left(\mathrm{mPEG}_{\mathrm{P}} \text { phen }\right)_{3}^{2+}$ complexes. As seen in Figure 2 inset, the calculated intensity distribution fits excellently to the experimental one. Alternatively, we also determined the distribution for the $\mathrm{Fe}(\mathrm{mPEG} \text {-phen })_{3}^{2+}$ complexes by calculating the probabilities of formation of tris-complexes with a particular chain length according to eq 2

$$
P_{n}=\sum_{i, j, k=19}^{38} P_{i} P_{j} P_{k}
$$

where $P_{n}$ is the probability of the formation of a complex which carries $n$ ethylene oxide repeat units altogether in the three ligands. $P_{i}, P_{j}$, and $P_{k}$ are the probabilities in the chain length distribution of $\mathrm{mPEG}_{-}$ phen, presented in Figure 1, with $i, j$, and $k$ repeat units and $i+j+k=n$.

The intensity distributions for the Fe(mPEG_phen) ${ }_{3}^{2+}$ complexes calculated by the two methods yield the same results. Based on the excellent coincidence of the measured and calculated distributions, it can be concluded that the formation of the Fe(mPEG_phen) ${ }_{3}^{2+}$ complex is independent of the mPEG_phen chain length, i.e., there is no significant difference in the binding energy of mPEG_phen of different sizes.

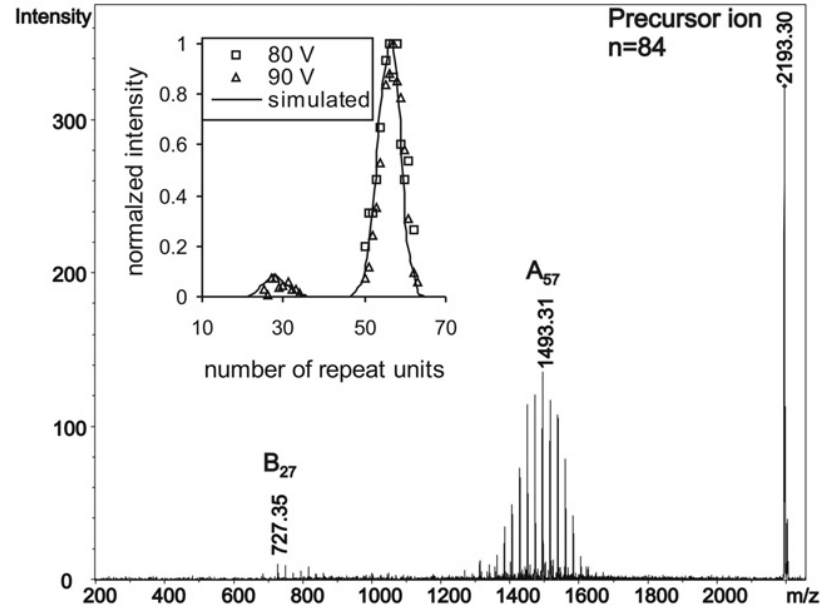

Figure 3. Product ion spectrum (MS/MS) of the Fe(mPEG phen $)_{3}^{2+}$ complex with a number of ethylene oxide units $84 \mathrm{(m} / \mathrm{z}$ 2193). The numbers in the subscripts represent the number of the ethylene oxide units. The inset shows the measured intensity distributions obtained at collision voltages, $80 \mathrm{~V}$ and $90 \mathrm{~V}$, together with the calculated intensity distribution of the product ions formed from the $\mathrm{Fe}\left(\mathrm{mPEG}\right.$-phen ${ }_{3}^{2+}$.

In further studies, the $\mathrm{Fe}\left(\mathrm{mPEG}\right.$ _phen ${ }_{3}^{2+}$ complex was subjected to collision-induced dissociation (CID). The product ion spectrum (MS/MS) of the Fe(mPEG_ phen $)_{3}^{2+}$ ion with a total number of repeat units 84 is shown in Figure 3.

As it can be seen in Figure 3, two series of peaks denoted by A and B appeared in the MS/MS spectrum. The $m / z$ difference between the adjacent peaks in each series is $\mathrm{m} / \mathrm{z} 22$; corresponding to the mass to charge ratio of an ethylene oxide unit bearing two charges. On the other hand, accurate mass measurements support that no cleavage of the backbone of the ligand takes place. Hence, the product ion series A and B are formed by the loss of one and two intact ligands from the precursor ion in a consecutive process under CID as depicted below:

$$
\begin{aligned}
& \mathrm{Fe}(\text { mPEG_phen })_{3}^{2+} \rightarrow \mathrm{Fe}(\text { mPEG_phen })_{2}^{2+} \\
& + \text { mPEG_phen } \\
& \mathrm{Fe}(\text { mPEG_phen })_{2}^{2+} \rightarrow \mathrm{Fe}(\text { mPEG_phen })^{2+} \\
& + \text { mPEG_phen }
\end{aligned}
$$

The product ions of series A reveal an intensity distribution centered at $\mathrm{m} / \mathrm{z} 1493$ corresponding to the bis-complex $\mathrm{Fe}$ (mPEG_phen) ${ }_{2}^{2+}$ with a total number of ethylene oxide units 57 . The occurrence of this series can be attributed to the process described by eq 3 , while the low intensity series $B$ is due to the consecutive dissociation of Fe(mPEG_phen) ${ }_{2}^{2+}$ (eq 4). It is important to clarify that although only one particular precursor complex ion was selected, the resulting product ion spectrum exhibits many peaks which may be associated with the distribution of mPEG_phen in the complex ion. 
For example, a tris-complex with 84 ethylene units altogether can be formed from mPEG_phen chains having 23, 28, and 33 units and/or bearing 27, 32, and 25 units, and so forth, generating lots of combinations. Thus, when a complex with a particular total number of repeat units is subjected to MS/MS, the resulting product ion distribution may be related to the mPEG_phen distribution.

To examine whether the loss of the ligand(s) is independent of the mPEG_phen chain-length, i.e., each fragmentation channel is completely equal, and how the distribution of series $\mathrm{A}$ and $\mathrm{B}$ are related to that of mPEG_phen (Figure 1), our computer simulation was extended to the dissociation of Fe(mPEG_phen $)_{3}^{2+}$. As seen in the inset of Figure 3, the simulated intensity distributions for both series fits quite well to the experimental ones, indicating that the dissociation of the $\mathrm{Fe}(\mathrm{mPEG} \text { _phen })_{3}^{2+}$ complex ion does not depend on the size of the ligand and the resulting intensity distributions of the product ions are related to that of $\mathrm{PPEG}_{-}$ phen. It can be also seen from Figure 3 inset that the collision voltage/energy does not affect the intensity distribution of the Fe(mPEG_Phen) $)_{2}^{2+}$ ions. The intensity distribution of Fe(mPEG_Phen) ${ }_{2}^{2+}$ recorded at $80 \mathrm{~V}$ collision voltage (near the dissociation threshold) matches that recorded at $90 \mathrm{~V}$ collision voltage. Therefore, the "kinetic shift" does not play a significant role in the dissociation of $\mathrm{Fe}\left(\mathrm{mPEG} \mathrm{P}_{-} \mathrm{Phen}\right)_{2}^{2+}$ into $\mathrm{Fe}\left(\mathrm{mPEG}_{-}\right.$ Phen $)^{2+}$ under our experimental conditions.

\section{Conclusions}

ESI-MS was proven to be capable of the investigation of the complex formation of $\mathrm{Fe}^{2+}$ with the polymer ligand mPEG_phen, and ESI-MS/MS can be used for studying the dissociation properties of these complexes in the gas phase. Based on our experiments and model calculations, it is concluded that both the formation and the collision-induced dissociation of the Fe(mPEG_phen $)_{3}^{2+}$ complex can be described in a simple way. Furthermore, such kind of ESI-MS and ESI-MS/MS investiga- tions can be extended to other complexes containing metal and polymer ligands, as well.

\section{Acknowledgments}

The authors acknowledge financial support for this work by grants K-72,524 and MU-00,204/2001 given by OTKA (National Scientific Research Fund, Hungary), grant GVOP-3.2.1.-2004-040152/3.0.

\section{Appendix}

\section{A. Supplementary Material}

Supplementary material associated with this article may be found in the online version at doi:10.1016/ j.jasms.2010.048.019.

\section{References}

1. Wong, S. F.; Meng, C. K.; Fenn, J. B. Multiple Charging in Electrospray Ionization of Poly(Ethylene Glycols). J. Phys. Chem. 1988, 92, 546-550.

2. Vachet, R. W.; Hartman J. R.; Gertner, J. W.; Callahan, J. H. Investigation of Metal Complex Coordination Structure Using Collision-Induced Dissociation and Ion-Molecule Reactions in a Quadrupole Ion Trap Mass Spectrometer. Int. J. Mass Spectrom. 2001, 204, 101-112.

3. Vachet, R. W.; Callahan, J. H. Quadrupole Ion Trap Studies of the Structure and Reactivity of Transition Metal Ion Pair Complexes. J. Mass Spectrom. 2000, 35, 311-320.

4. Molina-Svendsen, H.; Bojesen, G.; McKenzie, C. J. Gas-Phase Reactivity of Coordinatively Unsaturated Transition Metal Complex Ions Toward Molecular Oxygen. Inorg. Chem. 1998, 37, 1981-1983.

5. Tsierkezos, N. G.; Diefenbach, M.; Roithova, J.; Schroder, D.; Schwarz, H. Competitive Complexation of Gaseous Mn-II by 1,10-phenanthroline, 2,2'-bipyridine, and 4,5-diazafluorene. Inorg. Chem. 2005, 44, $4969-4978$.

6. Satterfield, M.; Brodbelt, J. S. Relative Binding Energies of Gas-Phase Pyridyl Ligand/Metal Complexes by Energy-Variable Collisionally Activated Dissociation in a Quadrupole Ion Trap. Inorg. Chem. 2001, 40, 5393-5400.

7. Forbes, M. W.; Volmer, D. A.; Francis, G. J.; Bohme, D. K. A Comparison of Data Analysis Methods for Determining Gas Phase Stabilities by CID: Alkali Metal Complexes of Polyether Ionophore Antibiotics. J. Am. Soc. Mass Spectrom. 2005, 16, 779-791.

8. Hayes, L. A.; Chappell, A. M.; Jellen, E. E.; Ryzhov, V. Binding of Metalloporphyrins to Model Nitrogen Bases: Collision-Induced Dissociation and Ion-Molecule Reaction Studies. Int. J. Mass Spectrom. 2003, $227,111-120$

9. Kéki, S.; Nagy, L.; Török, J.; Deák, Gy.; Zsuga, M. A Simple Method for Estimating Activation Energies Using the Fragmentation Yield: Collision-Induced Dissociation of Iron(II)-Phenanthroline Complexes in an Electrospray Ionization Mass Spectrometer. J. Am. Soc. Mass Spectrom. 2006, 17, 962-966

10. Kéki, S.; Nagy, L.; Deáak, Gy.; Zsuga, M. Multiple Charging of Poly(Propylene Glycol) by Binary Mixtures of Cations in Electrospray. J. Am. Soc. Mass Spectrom. 2005, 16, 152-157. 